\title{
Prevalence of mild cognitive impairment in community-dwelling Chinese populations aged over 55 years: a meta-analysis and systematic review
}

\author{
Yuan Lu $u^{1,2,3,4}$, Chaojie Liu ${ }^{2 *}$ (D), Dehua Yu ${ }^{1,3,4^{*}}$, Sally Fawkes ${ }^{2}$, Jia Ma ${ }^{3}$, Min Zhang ${ }^{3}$ and Chunbo Li ${ }^{5}$
}

\begin{abstract}
Background: Mild cognitive impairment (MCl) is an intermediate phase between normal cognitive ageing and overt dementia, with amnesic $\mathrm{MCl}(\mathrm{aMCl})$ being the dominant subtype. This study aims to synthesise the prevalence results of $\mathrm{MCl}$ and $\mathrm{aMCl}$ in community-dwelling populations in China through a meta-analysis and systematic review.

Methods: The study followed the Preferred Reporting Items for Systematic reviews and Meta-Analyses (PRISMA) protocol. English and Chinese studies published before 1 March 2020 were searched from ten electronic bibliographic databases. Two reviewers screened for relevance of the studies against the pre-defined inclusion and exclusion criteria and assessed the quality of the included studies using the Risk of Bias Tool independently. A random-effect model was adopted to estimate the prevalence of $\mathrm{MCl}$ and $\mathrm{aMCl}$, followed by sub-group analyses and meta-regression. Sensitivity and publication bias tests were performed to verify the robustness of the meta-analyses.

Results: A total of 41 studies with 112,632 participants were included in the meta-analyses. The Chinese communitydwelling populations over 55 years old had a pooled prevalence of 12.2\% [95\% confidence interval (Cl): 10.6, 14.2\%] for $\mathrm{MCl}$ and $10.9 \%[95 \% \mathrm{Cl}, 7.7,15.4 \%]$ for aMCl, respectively. The prevalence of $\mathrm{MCl}$ increased with age. The American Psychiatric Association's Diagnostic tool (DSM-IV) generated the highest MCI prevalence (13.5\%), followed by the Petersen criteria (12.9\%), and the National Institute on Aging Alzheimer's Association (NIA-AA) criteria (10.3\%). Women, rural residents, and those who lived alone and had low levels of education had higher $\mathrm{MCl}$ prevalence than others.

Conclusion: Higher $\mathrm{MCl}$ prevalence was identified in community-dwelling older adult populations in China compared with some other countries, possibly due to more broadened criteria being adopted for confirming the diagnosis. The study shows that $\mathrm{aMCl}$ accounts for $66.5 \%$ of $\mathrm{MCl}$, which is consistent with findings of studies undertaken elsewhere.
\end{abstract}

Systematic review registration number: PROSPERO CRD42019134686.

Keywords: Mild cognitive impairment, Prevalence, Systematic review, Meta-analysis

\footnotetext{
* Correspondence: c.liu@latrobe.edu.au; ydh1404@sina.com

${ }^{2} \mathrm{~S}$ chool of Psychology and Public Health, La Trobe University, Melbourne, VIC 3086, Australia

'Department of General Practice, Yangpu hospital, Tongji University School of Medicine, Shanghai 200090, China

Full list of author information is available at the end of the article
}

C C The Author(s). 2021 Open Access This article is licensed under a Creative Commons Attribution 4.0 International License, which permits use, sharing, adaptation, distribution and reproduction in any medium or format, as long as you give appropriate credit to the original author(s) and the source, provide a link to the Creative Commons licence, and indicate if changes were made. The images or other third party material in this article are included in the article's Creative Commons licence, unless indicated otherwise in a credit line to the material. If material is not included in the article's Creative Commons licence and your intended use is not permitted by statutory regulation or exceeds the permitted use, you will need to obtain permission directly from the copyright holder. To view a copy of this licence, visit http://creativecommons.org/licenses/by/4.0/ The Creative Commons Public Domain Dedication waiver (http://creativecommons.org/publicdomain/zero/1.0/) applies to the data made available in this article, unless otherwise stated in a credit line to the data. 


\section{Background}

The World Alzheimer Report 2016 [1] estimated that dementia is the third most serious health problem following cancer and cardio-cerebrovascular diseases, and costs the global economy around 315 billon US dollars annually. Like many other diseases, most of the burden of dementia is experienced by low- and middleincome countries [2]. China, as the most populated middle-income country, has attracted the greatest burden of dementia. About one quarter of people with a dementia diagnosis live in China [3]. The dementiaassociated disability and care burden in China is projected to be as high as US $\$ 250$ billion in 2020 , which accounts for nearly one fifth of the global costs associated with dementia [4].

Early intervention measures are considered to be the most cost-effective for managing dementia due to a lack of an effective treatment regimen [5]. Mild cognitive impairment (MCI) has been conceptualised as an intermediate phase between normal cognitive ageing and overt dementia [6]. MCI is a neurological disorder in older adults characterised by slight but noticeable deficits in memory and/or other thinking skills with minimal impacts on daily living functioning [7]. Some researchers argue that MCI represents an early stage of dementia [8], with a tendency of progressing into clinically diagnosed dementia at an annual rate around 30\% [9] and a lifetime rate of $60-90 \%$ [10].

MCI can be subcategorised into amnesic MCI (aMCI) and non-amnesic (naMCI). Memory loss is the predominant symptom of aMCI compared with naMCI which involves impairment in thinking skills other than memory [11]. Individuals with aMCI tend to progress into Alzheimer's disease $(\mathrm{AD})$; however, naMCI seems to represent a prodromal phase of frontotemporal dementia and dementia with Lewy bodies. Both aMCI and naMCI can lead to vascular dementia [12].

Internationally, extensive studies have been undertaken to determine the prevalence of $\mathrm{MCI}$, generating great variations in results. A systematic review published in 2012 reported a prevalence of MCI ranging from 0.5 to $42 \%$ in different countries and populations [13]. Recent studies in the US [14], Spain [15], Brazil [16], Saudi Arabia [17], and Japan [18] reported a range of MCI prevalence between 6.5 and $38.6 \%$. Significant variations in reported prevalence of MCI also exist within China. The Dementia Research Group reported a MCI prevalence of $0.8 \%$ in China [19], compared with $20.8 \%$ reported by the Chinese National Centre for Prevention and Control of Chronic and Non-communicable Diseases [20].

This study aims to determine the prevalence of MCI (including its subtypes) in community-dwelling older adults in China through a meta-analysis and systematic review. The study addresses several limitations of the existing systematic reviews [21, 22]. First, there is a need to carefully assess the representativeness of study samples. Inclusion of studies involving participants with certain special characteristics can seriously overestimate or underestimate the prevalence of MCI. For example, a study reported extremely high prevalence of MCI (74.23\%) in retired cadres, most in a very senior age [23]. By contrast, another study involving a high proportion of participants younger than 60 years reported only $2.4 \%$ of MCI [24]. Second, diagnostic criteria need to be considered in synthesising results. Applying different diagnostic tools and criteria is likely to lead to different results [25]. Many studies have failed to report specified criteria for confirmation of MCI [26]. Third, discrepancies in findings across study settings are common and they should not be mixed in synthesising analyses. MCI prevalence is usually higher in institutional settings than in communities [27]. To overcome the above-mentioned shortfalls, this study performed a series of subgroup analyses. To the best of our knowledge, no meta-analysis on aMCI prevalence in China has been reported. Findings of this study, especially those of the subgroup analyses, can provide a solid foundation for estimating MCI prevalence in community residents with different characteristics. This data is critical for planning preventive services in community settings.

\section{Methods}

We followed the Preferred Reporting Items for Systematic Reviews and Meta-Analyses (PRISMA) protocol (supplementary file 1), which delineates a four-phase flow diagram and a 27-item checklist (www.prisma-statement.org). The protocol of this systematic review was registered on PROSPERO and is available in full on the website https://www. crd.york.ac.uk/PROSPERO/display_record.php? ID=CRD42 019134686.

\section{Search strategy}

The well-established databases in English (Google Scholar, PubMed, Web of Science, Embase, CINAHL, PsycINFO) and Chinese languages (CQVIP, Wangfang, CNKI, Sinomed) were searched. All of the databases were searched from their inception to the $1^{\text {st }}$ of March 2020, using a combination of the following searching terms: ("mild cognitive impairment" or "cognitive dysfunction" or "early dementia") and (epidemiology, prevalence, rate, occurrence) (details provided in supplementary file 2). Hand searches were also performed to identify related papers through reference lists of the identified studies. A research librarian was consulted in developing the search strategy. The search results were exported to Endnote X9 (Thomson Reuters).

\section{Data extraction}

A total of 2136 studies were identified after deletion of duplications. Two reviewers (MZ and JM) screened the 
titles and abstracts of the articles and identified those that met the inclusion and exclusion criteria. The included articles had to fall into the category of original studies, including both population-based cross-sectional and longitudinal studies in community-based samples, with prevalence of $\mathrm{MCI}$ and/or aMCI as a primary study objective. The study samples were representative of community-dwelling older adults as indicated by the sampling strategy and did not include those admitted to long-term care facilities. MCI cases were identified using a MCI screening strategy followed by diagnostic confirmation. Since the pathobiological process in the human brain happens decades before the onset of dementia [28] and $\mathrm{MCI}$ screening may reasonably start at the age of 55 years, studies involving participants aged over 55 years were deemed eligible. There is a lack of consensus about when MCI screening should be started. Empirical evidence shows that the prevalence of MCI increases with age $[23,24]$. This study included participants $\geq 55$ years simply because there were no eligible MCI studies involving participants under 55 years old. In China, women and those engaging in labor-intensive jobs usually retire at the age of 55 years and are eligible for some preventive care packages delivered by community health services. These may include community MCI screening. Studies with a sample restricted to those with special characteristics such as disease condition (e.g. Parkinson disease, depression, stroke), occupation, internal migration, insurance, and literacy were excluded. Full texts of the eligible articles $(n=172)$ were then further assessed against the inclusion and exclusion criteria, and another 127 articles were excluded for failing to meet the inclusion criteria.

Two reviewers (MZ and JM) assessed the quality of the 45 studies that met the inclusion criteria by extracting key elements from the full texts into the Risk of Bias Tool [29]. The Risk of Bias Tool examines four aspects of external validity (target population representation, sample representation, random sampling, non-response bias), five aspects of internal validity (data collection proxy, acceptable case definition, instrument validity and reliability, data collection mode, appropriate parameter), and the overall risk of bias of the studies. This tool was designed for assessing bias in epidemiological surveys. The grading of the assessed aspects adopted the Cochrane Grades of Recommendation, Assessment, Development, and Evaluation (GRADE) scheme [30]. Each assessed aspect was given a rating of "low", "medium" or "high" risk of bias. Discrepancies between the two reviewers, if occurred, were resolved through discussions moderated by a third researcher. This resulted in a final sample of 41 studies without a high risk of bias for the final meta-analysis (Fig. 1).

Apart from the prevalence of MCI (including aMCI and naMCI), data in relation to study setting (urban/ rural), demographic characteristics (age, sex, educational attainments, living status) of participants, study period, screening tools, and diagnostic confirmation methods for each of the included studies were extracted. Empirical evidence shows that the prevalence of $\mathrm{MCI} / \mathrm{aMCI}$ is likely to vary by these factors [31].

\section{Statistical analysis}

MCI prevalence was the primary outcome of this metaanalysis. We synthesised the results for $\mathrm{MCI}$ in general as well as for aMCI specifically.

Publication bias of the included studies was assessed through visual symmetry of the funnel plots and Egger's tests [32]. A $p$ value lower than 0.05 indicates an absence of publication bias.

We performed heterogeneity analyses to determine the model used for meta-analyses. The $\mathrm{I}^{2}$ value was calculated and tested with Cochran Q tests. According to Cochrane Reviews [33], an $\mathrm{I}^{2}$ value of $0 \%$ indicates no observed heterogeneity, while a value greater than 25,50 and $75 \%$ indicates low, moderate, and high levels of heterogeneity, respectively. We chose a random effect model for meta-analyses and adopted sensitivity tests, meta-regression test and subgroup analyses strategies to handle high heterogeneity as suggested by Lipsey and Wilson [34]. The robustness of the meta-analyses was examined in sensitivity tests through sequentially removing each included study. The studies that deviated significantly from the others were excluded in the pooled results. Meta-regression was used to investigate available contributing factors on the heterogeneity. Subgroup meta-analyses were conducted whenever possible.

All statistical analyses were performed using Stata version 12.0 (Stata Corp, College Station, TX, USA). A two-sided $p<0.05$ was considered statistically significant. We also reported 95\% confidence intervals (CIs) for the results.

\section{Results \\ Characteristics of included studies}

The 41 eligible studies [19, 20, 24, 35-72] involved 112, 632 study participants, with MCI prevalence ranging from 1.21 to $33.03 \%$. The studies were conducted between 1998 and 2020. More than half (58\%) of the studies restricted participants from the age of over 60 years and $75 \%$ of the included studies had a sample size over 1000 participants. Most studies were cross-sectional, except for 7 longitudinal studies [39, 43, 46, 59, 61, 63, 66]. Sex composition varied across the studies, with women comprising 25.74 to $66.89 \%$ of study participants. About 30 neuropsychological test tools were used in the included studies of this systematic review. Of those tools, some test comprehensive cognitive function, such as CAMCOG (Cambridge Cognitive Examination) [73], CCAS (Chinese Cognitive Ability Scale) [74], CSI-D (Community 


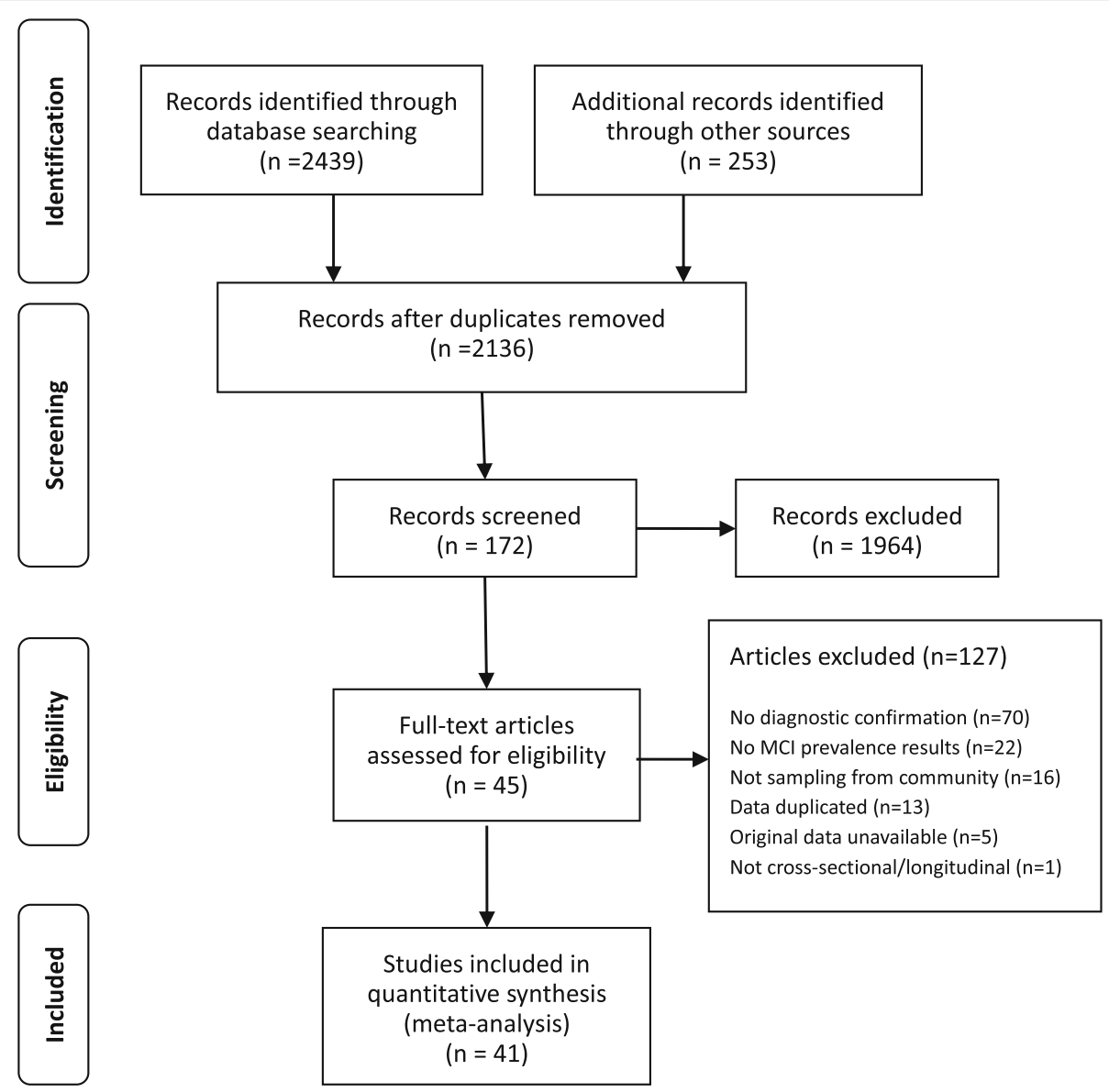

Fig. 1 the flowchart on the stages of including the studies in the systematic reviewed and meta-analysis (PRISMA 2009)

Screening Instrument for Dementia) [75], MMSE (MiniMental State Examination) [76], MoCA (the Montreal Cognitive Assessment) [77], NPI-Q (Neuropsychiatric Inventory Questionnaire) [78], OCST-E (Quick Cognitive Screening Scale) [79], SCID (Structured Clinical Interview for DSM-IV) [80], WAIS (Wechsler Adult Intelligence Scale) [81], WHO-BCAI (World Health OrganizationBattery of Cognitive Assessment Instrument) [82] and WHODAS-12 (12-item WHO Disability Assessment Schedule) [83], while others were adopted to test five cognitive domains: memory assessed by ALT (Associative Learning Test) [84], IMCT (Information-Memory-Concentration test) [85] and WMS-R (Wechsler Memory Scale-Revised) [86]; attention assessed by SDMT (Symbol Digit Modalities Test) [87] and STMT (Semantic Trail Making Test) [88]; vision tested by CDT (Clock drawing Test) [89] and ROCFR (Rey-Osterrieth Complex Figure Recall tests) [90]; language tested by AVLT (Auditory Verbal Learning Test) [91] and VFT (Verbal Fluency Test) [92]; and executive ability tested by ADL (Activity Daily Living) [93] and FAQ (Functional Activities Questionnaire) [94]. CDR (Clinical Dementia Rating Scale) [95], CESD (The Center for
Epidemiologic Studies Depression Scale) [96], DS (Digit Span) [97], HAMD (Hamilton Depression Rating Scale) [98], GDS (Global Deterioration Scale) [99], GMS (Geriatric Mental State) [100], SAS (Self-Rating Anxiety Scale) [101], and HIS (Hachinski Ischemic Index) [102] were used to exclude dementia and other mental disorder. MMSE [76] and the MoCA [77] scales were the predominant tools for MCI screening, supplemented by ADL [93] to test daily living function and other neurological tests such as CDR [95] to exclude dementia. The majority of the studies $(N=25)$ adopted Petersen's criteria for confirmation of diagnosis of MCI and aMCI, followed by the Diagnostic and the Statistical Manual of Mental Disorder 4th edition (DSM-IV) [80] developed by the American Psychiatric Association and the National Institute on Aging Alzheimer's Association (NIA-AA) [103] criteria (Table 1).

\section{Publication bias}

Most of the included studies were rated as having a moderate risk of bias, except for the four studies [104-107], which have a high risk of bias and were excluded from the final meta-analysis (Table 2). 
Table 1 Characteristics of studies $(n=41)$ included for meta-analysis

\begin{tabular}{|c|c|c|c|c|c|c|c|c|}
\hline $\begin{array}{l}\text { First } \\
\text { Author }\end{array}$ & $\begin{array}{l}\text { Study } \\
\text { period }\end{array}$ & $\begin{array}{l}\text { Sample } \\
\text { size }\end{array}$ & $\begin{array}{l}\text { Age } \\
\text { (years) }\end{array}$ & $\begin{array}{l}\text { Sex } \\
\text { (\% women) }\end{array}$ & $\begin{array}{l}\text { Study } \\
\text { location }\end{array}$ & $\begin{array}{l}\text { Diagnostic } \\
\text { criteria }\end{array}$ & Neurological tests & $\begin{array}{l}\mathrm{MCl} \text { prevalence } \\
(95 \% \mathrm{Cl})\end{array}$ \\
\hline Chen ND & 2012 & 465 & $\geq 60$ & $30.75 \%$ & Jiangsu & Petersen & ADL [93], CDT [89], SAS [101] & $\begin{array}{l}10.75 \% \\
(8.25-13.9 \%)\end{array}$ \\
\hline Ding D & 2011 & 2985 & $\geq 60$ & $54.22 \%$ & Shanghai & Petersen & $\begin{array}{l}\text { ADL [93], SAS [101], CDR [95], } \\
\text { HAMD [98], MMSE [76] }\end{array}$ & $\begin{array}{l}20.10 \% \\
(18.73-21.61 \%)\end{array}$ \\
\hline Guo GY & 2013 & 940 & $\geq 60$ & $56.81 \%$ & Hebei & Petersen & MoCA [77] & $\begin{array}{l}14.47 \% \\
(12.36-16.86 \%)\end{array}$ \\
\hline Guo X & 2011 & 1367 & $\geq 60$ & $50.40 \%$ & Hunan & DSM-IV & $\begin{array}{l}\text { CDR [95], GDS [99], } \\
\text { HIS [102], IMCT [85], } \\
\text { MMSE [76] }\end{array}$ & $\begin{array}{l}10.17 \% \\
(8.68-11.88 \%)\end{array}$ \\
\hline Hai S & 2007 & 202 & $\geq 80$ & $25.74 \%$ & Sichuan & Petersen & $\begin{array}{l}\text { ADL [93], CAMCOG [73], } \\
\text { GDS [99], MMSE [76] }\end{array}$ & $\begin{array}{l}30.2 \% \\
(24.28-36.85 \%)\end{array}$ \\
\hline $\mathrm{He} L$ & 2014 & 842 & $\geq 60$ & $48.81 \%$ & Jiangxi & DSM-IV & $\begin{array}{l}\text { ADL [93], CDR [95], GDS [99], } \\
\text { HIS [102], MoCA [77] }\end{array}$ & $\begin{array}{l}13.42 \% \\
(11.28-15.89 \%)\end{array}$ \\
\hline $\mathrm{Hu} R$ & 2009 & 5887 & $\geq 55$ & $56.38 \%$ & Mongolia & DSM-IV & $\begin{array}{l}\text { ADL [93], CDR [95], HIS [102], } \\
\text { HAMD [98], MMSE [76], } \\
\text { MoCA [77] }\end{array}$ & $\begin{array}{l}20.60 \% \\
(19.59-21.66 \%)\end{array}$ \\
\hline Huang R & 2002 & 4697 & $\geq 60$ & $58.85 \%$ & Guangzhou & Petersen & $\begin{array}{l}\text { ADL [93], CDR [95], CESD [96], } \\
\text { HAMD [98], MMSE [76] }\end{array}$ & $\begin{array}{l}5.47 \% \\
(4.86-6.16 \%)\end{array}$ \\
\hline Jia J & 2009 & 10,276 & $\geq 65$ & N/A & National & Petersen & $\begin{array}{l}\text { AVLT, CDR [95], CDT [89], } \\
\text { CESD [96], FAQ [94], HIS, } \\
\text { MoCA [77], MMSE [76], } \\
\text { STMT [88], VFT [92], AVLT [91] }\end{array}$ & $\begin{array}{l}20.8 \% \\
(20.02-21.59 \%)\end{array}$ \\
\hline Jiang $\sqcup J$ & 2016 & 895 & $\geq 60$ & $51.06 \%$ & Jilin & NIA-AA & $\begin{array}{l}\text { ADL [93], CDR [95], GDS [99], } \\
\text { HIS [102], MMSE [76], } \\
\text { MoCA [77] }\end{array}$ & $\begin{array}{l}5.36 \% \\
(4.07-7.04 \%)\end{array}$ \\
\hline Lao ML & 2010 & 7665 & $\geq 55$ & $54.22 \%$ & Hainan & Petersen & $\begin{array}{l}\text { ADL [93], GDS [99], } \\
\text { MMSE [76] }\end{array}$ & $\begin{array}{l}4.25 \% \\
(3.82-4.73 \%)\end{array}$ \\
\hline Li CP & 2014 & 1971 & $\geq 60$ & $62.61 \%$ & Shandong & DSM-IV & $\begin{array}{l}\text { ADL [93], CDR [95], GDS [99], } \\
\text { HIS [102], MMSE [76] }\end{array}$ & $\begin{array}{l}33.03 \% \\
(30.99-35.14 \%)\end{array}$ \\
\hline LiW & 2019 & 3246 & $\geq 60$ & N/A & Shanghai & Petersen & $\begin{array}{l}\text { ALT [84], AVLT [91], DS [97], } \\
\text { MMSE [76], MoCA [77], } \\
\text { NPI-Q [78], VFT [92], WAIS [81] }\end{array}$ & $\begin{array}{l}17.07 \%^{*} \\
(15.79-18.42 \%)\end{array}$ \\
\hline LiX & 2013 & 1020 & $\geq 55$ & $63.33 \%$ & Beijing & Petersen & $\begin{array}{l}\text { AVLT [91], CDT [89], } \\
\text { MMSE [76], ROCFR [90], } \\
\text { SDMT [87], STMT [88] }\end{array}$ & $\begin{array}{l}15.69 \% \\
(13.58-18.05 \%)\end{array}$ \\
\hline Liao B & 2012 & 399 & $\geq 60$ & $53.63 \%$ & Jiangxi & Petersen & ADL [93], HIS [102], MoCA [77] & $\begin{array}{l}10.28 \% \\
(7.67-13.64 \%)\end{array}$ \\
\hline Liu $\mathrm{H}$ & 2018 & 1796 & $\geq 60$ & $53.95 \%$ & Shanghai & DSM-IV & $\begin{array}{l}\text { ADL [93], GDS [99], HIS [102], } \\
\text { MoCA [77] }\end{array}$ & $\begin{array}{l}17.65 \% \\
(15.96-19.48 \%)\end{array}$ \\
\hline $\mathrm{MaF}$ & 2016 & 5067 & $\geq 65$ & $57.80 \%$ & Tianjin & Petersen & ADL [93], MMSE [76], WAIS [81] & $\begin{array}{l}11.33 \% \\
(10.48-12.23 \%)\end{array}$ \\
\hline Meng WQ & 2009 & 5452 & $\geq 55$ & $53.62 \%$ & Inner Mongolia & Petersen & ADL [93], MMSE [76] & $\begin{array}{l}22.50 \% \\
(21.16-23.37 \%)\end{array}$ \\
\hline Pan ZD & 2012 & 300 & $\geq 60$ & $57.14 \%$ & Shanghai & Petersen & $\begin{array}{l}\text { ADL [93], CDR [95], GDS [99], } \\
\text { HIS [102], MMSE [76], } \\
\text { MoCA [77] }\end{array}$ & $\begin{array}{l}22.33 \% \\
(17.99-27.38 \%)\end{array}$ \\
\hline Qin $\mathrm{HY}$ & 2012 & 4086 & $\geq 55$ & $65.00 \%$ & Shanghai & Petersen & $\begin{array}{l}\text { ADL [93], CDR [95], } \\
\text { MMSE [76] }\end{array}$ & $\begin{array}{l}14.98 \% \\
(13.92-16.11 \%)\end{array}$ \\
\hline Qiu CJ & 2001 & 3910 & $\geq 55$ & $50.82 \%$ & Chengdu & Petersen & $\begin{array}{l}\text { CDR [95], CESD [96], } \\
\text { MMSE [76] }\end{array}$ & $\begin{array}{l}2.35 \% \\
(1.92-2.88 \%)\end{array}$ \\
\hline Rao DP & 2009 & 2111 & $\geq 65$ & $59.50 \%$ & Guangzhou & Petersen & $\begin{array}{l}\text { ADL [93], CDR [95], GDS [99], } \\
\text { MMSE [76], MoCA [77] }\end{array}$ & $\begin{array}{l}14.16 \% \\
(12.74-15.72 \%)\end{array}$ \\
\hline Ren CF & 2011 & 946 & $\geq 60$ & $49.26 \%$ & Jiangxi & DSM-IV & $\begin{array}{l}\text { ADL [93], CDR [95], GDS [99], } \\
\text { HIS [102], MoCA [77] }\end{array}$ & $\begin{array}{l}10.47 \% \\
(8.67-12.58 \%)\end{array}$ \\
\hline
\end{tabular}


Table 1 Characteristics of studies $(n=41)$ included for meta-analysis (Continued)

\begin{tabular}{|c|c|c|c|c|c|c|c|c|}
\hline $\begin{array}{l}\text { First } \\
\text { Author }\end{array}$ & $\begin{array}{l}\text { Study } \\
\text { period }\end{array}$ & $\begin{array}{l}\text { Sample } \\
\text { size }\end{array}$ & $\begin{array}{l}\text { Age } \\
\text { (years) }\end{array}$ & $\begin{array}{l}\text { Sex } \\
\text { (\% women) }\end{array}$ & $\begin{array}{l}\text { Study } \\
\text { location }\end{array}$ & $\begin{array}{l}\text { Diagnostic } \\
\text { criteria }\end{array}$ & Neurological tests & $\begin{array}{l}\mathrm{MCl} \text { prevalence } \\
(95 \% \mathrm{Cl})\end{array}$ \\
\hline Song XZ & 2011 & 2279 & $\geq 60$ & $51.21 \%$ & Guangzhou & Petersen & $\begin{array}{l}\text { ADL [93], CDR [95], GDS [99], } \\
\text { HIS [102], HAMD [98], } \\
\text { MMSE [76] }\end{array}$ & $\begin{array}{l}7.33 \% \\
(6.33-8.47 \%)\end{array}$ \\
\hline Sosa AL & 2007 & 2014 & $\geq 65$ & $63.33 \%$ & National & DSM-IV & $\begin{array}{l}\text { CSI-D [75], GMS [100], } \\
\text { NPI-Q [78], WHODAS-12 }\end{array}$ & $\begin{array}{l}7.99 \%^{*} \\
(6.89-9.26 \%)\end{array}$ \\
\hline Su C & 2011 & 341 & $\geq 60$ & $52.49 \%$ & Guangzhou & Petersen & $\begin{array}{l}\text { ADL [93], CDR [95], GDS [99], } \\
\text { WHO-BCAI [82], MMSE [76], } \\
\text { MoCA [77] }\end{array}$ & $\begin{array}{l}12.32 \% \\
(9.24-16.23 \%)\end{array}$ \\
\hline Sun $Y$ & 2013 & 10,432 & $\geq 65$ & $52.32 \%$ & Taiwan & NIA-AA & ADL [93], CDR [95], MMSE [76] & $\begin{array}{l}19.64 \% \\
(18.89-20.41 \%)\end{array}$ \\
\hline Tang MN & 1998 & 5385 & $\geq 55$ & N/A & Chengdu & DSM-III & $\begin{array}{l}\text { ADL [93], CDR [95], CESD [96], } \\
\text { HIS [102], HAMD [98], } \\
\text { MMSE [76] }\end{array}$ & $\begin{array}{l}1.21 \% \\
(0.95-1.54 \%)\end{array}$ \\
\hline Tang Z & 2004 & 1865 & $\geq 60$ & $51.90 \%$ & Beijing & Petersen & $\begin{array}{l}\text { ADL, CDR [95], CESD [96], } \\
\text { MMSE [76] }\end{array}$ & $\begin{array}{l}11.64 \% \\
(10.26-13.17 \%)\end{array}$ \\
\hline Wang $T$ & 2012 & 1005 & $\geq 60$ & N/A & Shanghai & DSM-IV & $\begin{array}{l}\text { ADL [93], AVLT [91], CDR [95], } \\
\text { DS [97], GDS [99], HIS [102], } \\
\text { MMSE [76], MoCA [77], } \\
\text { WMS-R [86] }\end{array}$ & $\begin{array}{l}22.29 \%^{*} \\
(19.82-24.96 \%)\end{array}$ \\
\hline Wang TT & 2017 & 1781 & $\geq 60$ & $60.47 \%$ & Chongqing & Petersen & ADL [93], GDS, MMSE [76] & $\begin{array}{l}11.73 \% \\
(10.32-13.31 \%)\end{array}$ \\
\hline Wang YP & 2009 & 6152 & $\geq 65$ & N/A & Shanxi & DSM-IV & $\begin{array}{l}\text { CDR [95], MMSE [76], } \\
\text { WHO-BCAI [82] }\end{array}$ & $\begin{array}{l}9.75 \% \\
(9.04-10.52 \%)\end{array}$ \\
\hline Wang ZZ & 2013 & 689 & $\geq 55$ & $62.70 \%$ & Ningxia & $\begin{array}{l}\text { Chinese } \\
\text { Dementia } \\
\text { guideline }\end{array}$ & ADL [93], GDS [99], MMSE [76] & $\begin{array}{l}18.29 \% \\
(15.58-21.35 \%)\end{array}$ \\
\hline Wu Y & 2014 & 1846 & $\geq 60$ & $53.36 \%$ & Jiangsu & Petersen & $\begin{array}{l}\text { CCAS [74], CDR [95], HAMD [98], } \\
\text { MMSE [76], QCST-E [79] }\end{array}$ & $\begin{array}{l}17.17 \% \\
(15.52-18.96 \%)\end{array}$ \\
\hline Xiao SF & 2016 & 1068 & $\geq 60$ & N/A & Shanghai & Petersen & $\begin{array}{l}\text { AVLT [91], MMSE [76], } \\
\text { MoCA [77], WMS-R [86], } \\
\text { WHO-BCAI [82] }\end{array}$ & $\begin{array}{l}25.00 \% \\
(22.50-27.68 \%)\end{array}$ \\
\hline Xu SJ & 2011 & 2426 & $\geq 60$ & $60.68 \%$ & Hebei & Petersen & $\begin{array}{l}\text { ADL [93], GDS [99], MMSE [76], } \\
\text { MoCA [77], SAS [101] }\end{array}$ & $\begin{array}{l}21.68 \% \\
(20.09-23.37 \%)\end{array}$ \\
\hline Yin LY & 2009 & 1011 & $\geq 65$ & $59.45 \%$ & Hebei & Petersen & $\begin{array}{l}\text { CDR [95], CESD [96], FAQ, } \\
\text { GDS [99], MMSE [76], } \\
\text { MoCA [77] }\end{array}$ & $\begin{array}{l}6.63 \% \\
(5.25-8.33 \%)\end{array}$ \\
\hline Yuan J & 2010 & 3311 & $\geq 60$ & $66.89 \%$ & Shanghai & Petersen & HIS [102], SCID-I/P [80] & $\begin{array}{l}19.06 \% \\
(17.76-20.43 \%)\end{array}$ \\
\hline Zhang XQ & 2012 & 1764 & $\geq 60$ & $55.95 \%$ & Changsha & Petersen & $\begin{array}{l}\text { ADL [93], CDR [95], GDS, } \\
\text { MMSE [76], MoCA [77] }\end{array}$ & $\begin{array}{l}16.27 \% \\
(14.62-18.07 \%)\end{array}$ \\
\hline Zhou DS & 2010 & 1227 & $\geq 60$ & $56.32 \%$ & Zhejiang & DSM-IV & $\begin{array}{l}\text { CDR [95], CESD [96], GDS [99], } \\
\text { HIS [102], } \\
\text { IMCT [85], MMSE [76] }\end{array}$ & $\begin{array}{l}8.72 \% \\
(7.27-10.43 \%)\end{array}$ \\
\hline Zhu XQ & 2008 & 1511 & $\geq 60$ & $54.60 \%$ & Xinjiang & DSM-IV & $\begin{array}{l}\text { CDR [95], GD S [99], HIS [102], } \\
\text { HAMD [98], MMSE [76] }\end{array}$ & $\begin{array}{l}9.79 \% \\
(8.40-11.40 \%)\end{array}$ \\
\hline
\end{tabular}

Note: * aMCI prevalence

\section{Robust tests for pooled results}

High levels of heterogeneity were found $\left(\mathrm{I}^{2}>75 \%\right)$ across the 41 included studies. Of the 38 studies reporting MCI prevalence, three $[39,45,59]$ showed significant deviation from the others both in sensitivity tests and visual funnel asymmetry. The Egger's and Begg's tests also revealed significant publication bias in the studies $(\beta=$ 0.002, $p<0.01$ ). Cohort effects could explain $20.75 \%$ of heterogeneity from meta-regression test. Further subgroup analyses on MCI prevalence were warranted as no significant associations $(p>0.05)$ between the prevalence of MCI and other two potential bias factors were found in the meta-regression analyses (Table 3 ).

Of the 8 studies $[19,20,36,46,47,50,54,61]$ reporting aMCI prevalence, no study showed significant deviation from the others in sensitivity tests. The Egger's 
Table 2 Risk of bias of included studies $(n=45)$

\begin{tabular}{|c|c|c|c|c|}
\hline No. & Study & External validity & Internal validity & Overall \\
\hline 1 & Chen ND, 2012 & Moderate Risk & Moderate Risk & Moderate Risk \\
\hline 2 & Ding D, 2015 & Low Risk & Low Risk & Low Risk \\
\hline 3 & Guo GY, 2013 & Moderate Risk & Moderate Risk & Moderate Risk \\
\hline 4 & Guo XY, 2013 & Moderate Risk & Moderate Risk & Moderate Risk \\
\hline 5 & Hai S, 2011 & Moderate Risk & Moderate Risk & Moderate Risk \\
\hline 6 & He L, 2015 & Low Risk & Moderate Risk & Moderate Risk \\
\hline 7 & Hu R, 2012 & Moderate Risk & Moderate Risk & Moderate Risk \\
\hline 8 & Huang R, 2008 & Low Risk & Low Risk & Low Risk \\
\hline 9 & JIA J, 2013 & Low Risk & Low Risk & Low Risk \\
\hline 10 & Jiang LJ, 2017 & Moderate Risk & Moderate Risk & Moderate Risk \\
\hline 11 & Lao ML, 2011 & Moderate Risk & Moderate Risk & Moderate Risk \\
\hline 12 & Li CP, 2014 & low Risk & Moderate Risk & Moderate Risk \\
\hline 13 & Li X, 2013 & Moderate Risk & Moderate Risk & Moderate Risk \\
\hline 14 & Li W, 2020 & low Risk & Low Risk & Low Risk \\
\hline 15 & Liao B, 2012 & Moderate Risk & Moderate Risk & Moderate Risk \\
\hline 16 & Liu H, 2018 & low Risk & Moderate Risk & Moderate Risk \\
\hline 17 & Ma F, 2016 & Low Risk & Low Risk & Low Risk \\
\hline 18 & Meng WQ, 2010 & Moderate Risk & Moderate Risk & Moderate Risk \\
\hline 19 & Pan HY, 2012 & Moderate Risk & High Risk & High Risk \\
\hline 20 & Pan ZD, 2012 & Low Risk & Moderate Risk & Moderate Risk \\
\hline 21 & Peng Z, 2019 & Moderate Risk & High Risk & High Risk \\
\hline 22 & Qin HY, 2014 & Low Risk & Low Risk & Low Risk \\
\hline 23 & Qiu CJ, 2003 & Moderate Risk & Low Risk & Moderate Risk \\
\hline 24 & Rao D, 2018 & Low Risk & Low Risk & Low Risk \\
\hline 25 & Ren CF, 2013 & Moderate Risk & Low Risk & Moderate Risk \\
\hline 26 & Song XZ, 2012 & Low Risk & Moderate Risk & Moderate Risk \\
\hline 27 & Sosa AL, 2012 & Moderate Risk & Moderate Risk & Moderate Risk \\
\hline 28 & Su C, 2013 & Moderate Risk & Moderate Risk & Moderate Risk \\
\hline 29 & Sun Y, 2014 & Low Risk & Moderate Risk & Moderate Risk \\
\hline 30 & Tang Z, 2007 & Low Risk & Moderate Risk & Moderate Risk \\
\hline 31 & Tang MN, 2000 & Low Risk & Moderate Risk & Moderate Risk \\
\hline 32 & Wang T, 2017 & Low Risk & Moderate Risk & Moderate Risk \\
\hline 33 & Wang TT, 2017 & Low Risk & Low Risk & Low Risk \\
\hline 34 & Wang YP, 2011 & Moderate Risk & Moderate Risk & Moderate Risk \\
\hline 35 & Wang ZZ, 2013 & Moderate Risk & Moderate Risk & Moderate Risk \\
\hline 36 & Wu L, 2016 & Moderate Risk & High Risk & High Risk \\
\hline 37 & Wu Y, 2017 & Low Risk & Moderate Risk & Moderate Risk \\
\hline 38 & Xiao SF, 2016 & Low Risk & Low Risk & Low Risk \\
\hline 39 & Xu SJ, 2014 & Low Risk & Moderate Risk & Moderate Risk \\
\hline 40 & Yin LY, 2010 & Low Risk & Moderate Risk & Moderate Risk \\
\hline 41 & Yuan J, 2013 & Moderate Risk & Moderate Risk & Moderate Risk \\
\hline 42 & Zhang XQ, 2014 & Low Risk & Low Risk & Low Risk \\
\hline 43 & Zhong SY, 2018 & Moderate Risk & High Risk & High Risk \\
\hline 44 & Zhou DS, 2011 & Moderate Risk & Low Risk & Moderate Risk \\
\hline 45 & Zhu XQ, 2009 & Moderate Risk & Low Risk & Moderate Risk \\
\hline
\end{tabular}


Table 3 Meta-regression analyses result

\begin{tabular}{lllllll}
\hline Inhr & Coef. & Std. Err. & $\mathbf{t}$ & $\mathbf{P}>\mathbf{| t |}$ & [95\% Conf. Interval] \\
\hline Age & .2187126 & .1562825 & 1.40 & 0.171 & -.0988917 & .5363169 \\
Study period & .3282157 & .1214176 & 2.70 & 0.011 & .0814654 & .574966 \\
Diagnostic criteria & -.1795994 & .1203679 & -1.49 & 0.145 & -.4242164 & .0650176 \\
Constant & -3.146882 & .5048756 & -6.23 & 0.000 & -4.172913 & -2.120851 \\
\hline
\end{tabular}

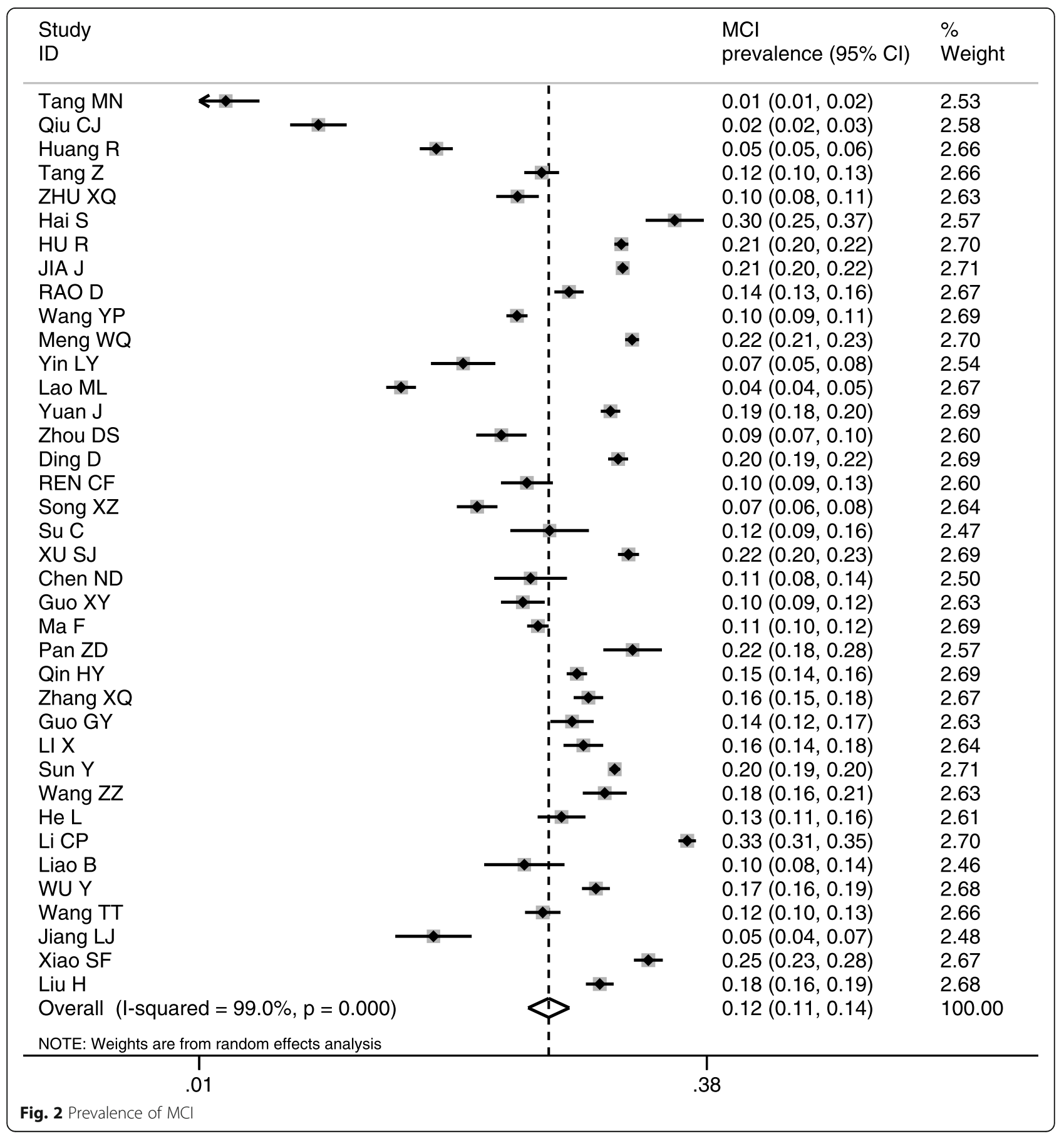




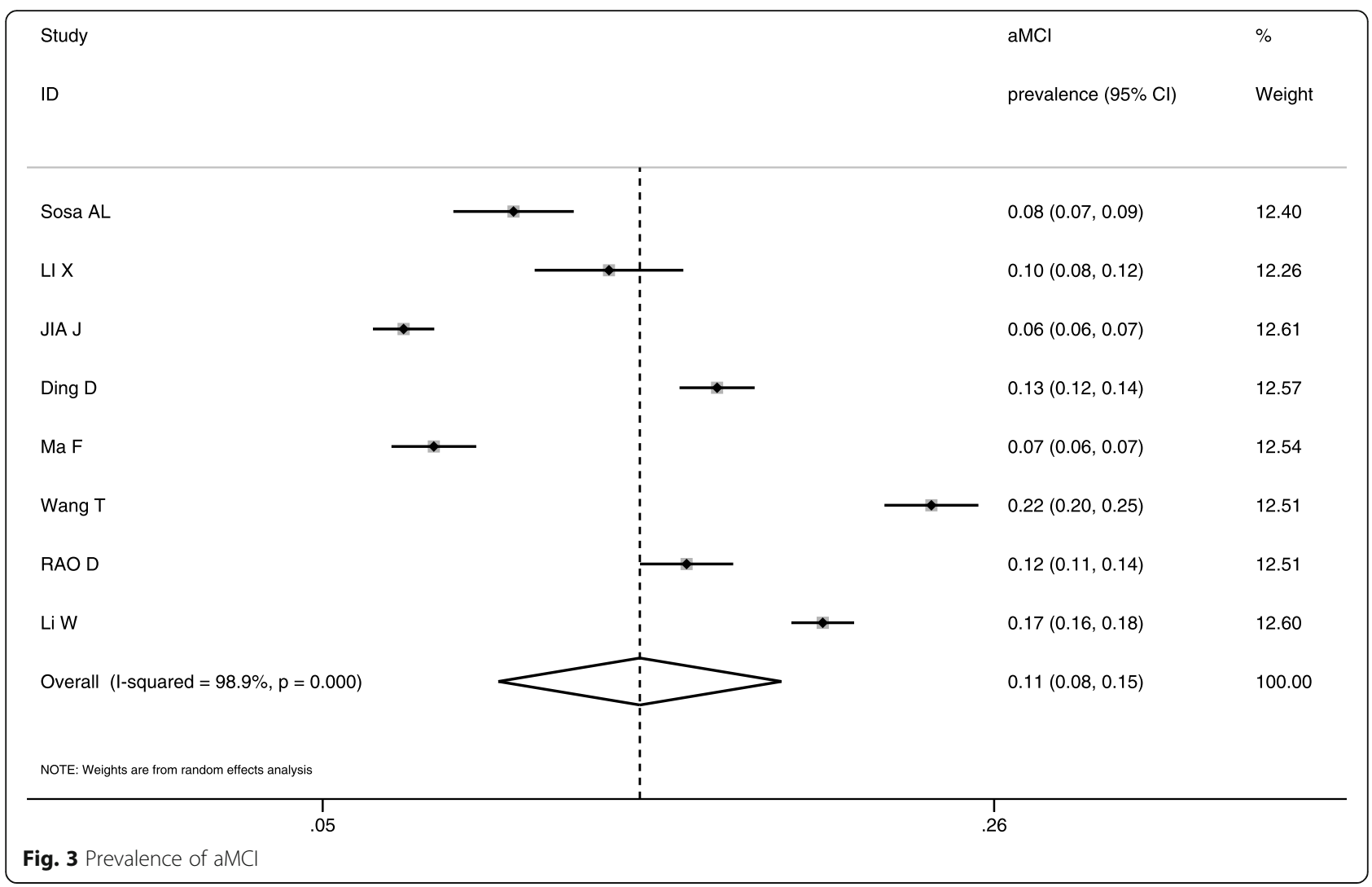

and Begg's tests revealed no significant publication bias either $(\beta=1.0, p=0.902)$.

\section{Prevalence of $\mathrm{MCl}$ and $\mathrm{aMCl}$ - results of meta-analyses}

The meta-analysis of the 38 studies $(n=106,367)$ with a random-effect estimate resulted in a MCI prevalence of $12.2 \%$ [95\% confidence interval (CI): 10.6, 14.2\%] (Fig. 2).

The meta-analysis of the 8 studies $(n=27,613)$ generated a result of $10.9 \%$ prevalence of aMCI [95\%CI: 7.7, 15.4\%] (Fig. 3).

\section{Results of subgroup analyses}

Heterogeneity of the subgroup analyses reduced significantly. The prevalence of MCI increased with age: $7.6 \%$ for $55-59$ years; $9.5 \%$ for $60-69$ years; $14.6 \%$ for $70-79$ years; and $23.6 \%$ for 80 years and older. Women had a higher prevalence of MCI than men. Those who resided in rural areas, lived alone, and had lower educational attainments had higher MCI prevalence than others. The DSM-IV diagnostic criteria generated the highest MCI prevalence (13.5\%), compared with $12.9 \%$ using the Petersen criteria and $10.3 \%$ using the NIA-AA diagnosis. The four studies [24, 42, 59, 60] conducted before 2005 reported significantly lower prevalence of MCI than those after 2005 (Table 4).

\section{Discussion}

\section{Prevalence of $\mathrm{MCl}$ and $\mathrm{aMCl}$}

Overall, $12.2 \%$ of Chinese community-dwelling older adults have MCI. This result is consistent with findings of previous systematic reviews [21,22] although they adopted much more broadened standards in terms of diagnostic confirmation and inclusion/exclusion criteria for included studies. If we exclude participants younger than 60 years in this systematic review, the result would be comparable to the MCI prevalence levels (12.7 to $14.7 \%)$ revealed in those systematic reviews. Similar levels of MCI prevalence were also reported in the 65 years and older populations in Greece [108] and Georgia [109]. Such a level is high compared to the studies conducted in other populations where more strict diagnostic criteria were adopted (e.g. neuropsychological scores at least 1.5 standard deviations below the adjusted norm). For example, a prevalence proportion of $5.3 \%$ for $\mathrm{MCI}$ was found in Finland in community residents aged between 60 and 76 years [110]. An Italian study found a prevalence proportion of $4.9 \%$ for $\mathrm{MCI}$ in communitydwelling residents older than 65 years [111]. It is likely that a higher percentage of older adults may have lived in aged care institutions in these developed nations. However, this does not offer a full explanation of the low prevalence of MCI. Lower levels of MCI prevalence were also found in some developing nations, such as 
Table 4 Subgroup meta-analyses on the prevalence of $\mathrm{MCl}$

\begin{tabular}{|c|c|c|c|c|c|c|}
\hline \multirow[t]{2}{*}{ Subgroup } & \multirow{2}{*}{$\begin{array}{l}\text { Included } \\
\text { studies }\end{array}$} & \multirow{2}{*}{$\begin{array}{l}\text { Study participants } \\
\text { (sample size) }\end{array}$} & \multicolumn{2}{|c|}{ Random-effect Model } & \multicolumn{2}{|c|}{ Heterogeneity } \\
\hline & & & $\begin{array}{l}\mathrm{MCl} \text { Prevalence } \\
(95 \% \mathrm{Cl})\end{array}$ & $p$ & $\overline{1^{2}}$ & $\mathrm{Ph}$ \\
\hline \multicolumn{7}{|l|}{ Age (Years) } \\
\hline $55-59$ & 3 & 5951 & $0.076(0.025-0.226)$ & $<0.001$ & $99.2 \%$ & 0.922 \\
\hline $60-69$ & 24 & 23,095 & 0.095(0.074-0.121) & $<0.001$ & $97.8 \%$ & 0.356 \\
\hline $70-79$ & 25 & 22,902 & $0.146(0.124-0.171)$ & $<0.001$ & $96.6 \%$ & 0.153 \\
\hline$\geq 80$ & 25 & 9397 & $0.236(0.204-0.274)$ & $<0.001$ & $93.5 \%$ & 0.122 \\
\hline \multicolumn{7}{|l|}{ Sex } \\
\hline Men & 34 & 45,609 & $0.115(0.097-0.136)$ & $<0.001$ & $97.4 \%$ & 0.233 \\
\hline Women & 34 & 36,027 & $0.138(0.117-0.163)$ & $<0.001$ & $98.3 \%$ & 0.224 \\
\hline \multicolumn{7}{|l|}{ Residency } \\
\hline Urban & 32 & 71,801 & $0.114(0.098-0.132)$ & $<0.001$ & $98.4 \%$ & 0.174 \\
\hline Rural & 12 & 25,137 & $0.136(0.106-0.176)$ & $<0.001$ & $98.8 \%$ & 0.193 \\
\hline \multicolumn{7}{|l|}{ Living status } \\
\hline With family & 10 & 13,941 & $0.141(0.110-0.182)$ & $<0.001$ & $97.6 \%$ & 0.157 \\
\hline alone & 10 & 3518 & $0.182(0.136-0.244)$ & $<0.001$ & $95.0 \%$ & 0.206 \\
\hline \multicolumn{7}{|c|}{ Education attainment } \\
\hline$<$ Primary school & 18 & 10,974 & $0.172(0.122-0.243)$ & $<0.001$ & $98.3 \%$ & 0.540 \\
\hline Primary school & 18 & 14,502 & $0.120(0.083-0.174)$ & $<0.001$ & $98.5 \%$ & 0.623 \\
\hline Middle school & 21 & 11,367 & $0.091(0.072-0.115)$ & $<0.001$ & $94.3 \%$ & 0.418 \\
\hline$\geq$ High school & 21 & 9568 & $0.063(0.046-0.085)$ & $<0.001$ & $94.2 \%$ & 0.515 \\
\hline \multicolumn{7}{|l|}{ Diagnostic criteria } \\
\hline Peterson & 25 & 67,267 & $0.129(0.107-0.154)$ & $<0.001$ & $98.9 \%$ & 0.209 \\
\hline DSM-IV & 9 & 21,699 & $0.135(0.097-0.188)$ & $<0.001$ & $99.0 \%$ & 0.257 \\
\hline NIA-AA & 2 & 11,327 & $0.103(0.029-0.369)$ & $<0.001$ & $98.8 \%$ & 0.833 \\
\hline \multicolumn{7}{|l|}{ Study period } \\
\hline$<2005$ & 4 & 15,857 & $0.037(0.016-0.087)$ & $<0.001$ & $99.2 \%$ & 0.769 \\
\hline$\geq 2005$ & 30 & 90,510 & $0.141(0.124-0.160)$ & $<0.001$ & $98.6 \%$ & 0.142 \\
\hline
\end{tabular}

6.45\% in Mexico [112] and 6.10\% in Brazil [16] from community residents over 60 years despite the fact that most studies in these countries used the Petersen criteria, the same as the included studies in this current systematic review.

This study included participants aged between 55 and 59 years in the meta-analyses. To our knowledge, this is the first attempt to estimate MCI prevalence in people younger than 60 years. Indeed, there is a dramatic increase in MCI prevalence in the community residents older than 60 years as revealed in this study. However, $7.6 \%$ of those at the age between 55 and 59 years were still diagnosed with MCI. This indicates a potential benefit of starting MCI screening in this group of population. Many preventive care packages have been designed for people older than 55 years in community health services, which present an opportunity for introducing MCI screening services. But before such a policy is developed, robust studies into the cost benefits of such services are needed. Currently, there are few studies of MCI in people younger than 60 years. Only eight studies [24, 41, 44, 47, 51, 53, 59, 64] were identified in this systematic review. Nevertheless, the pathobiological process in the human brain happens decades before the onset of dementia [28] and MCI screening may reasonably start at the age of 55 years.

It is evident that aMCI is the predominant form of MCI in Chinese populations. This study estimated that $10.9 \%$ community-dwelling Chinese populations older than 55 years have aMCI, higher than those reported in most international studies $[113,114]$. This study found that aMCI account for $66.5 \%$ of all MCI cases. Internationally, aMCI as a percentage of $\mathrm{MCI}$ ranges between 30 and $77 \%$. The lowest prevalence of aMCI (2.4\%) was 
reported in Mexico [112], while the highest (around 11\%) was reported by the Mayo Clinic Study of Aging from Olmsted County, USA, residents between 70 and 89 years old [115].

\section{Factors associated with the prevalence of $\mathrm{MCl}$ and $\mathrm{aMCl}$}

High levels of heterogeneity are evident in the studies included in our meta-analyses. Many factors may have contributed to the variations of findings within individual studies. MCI prevalence varies by diagnostic tools, study settings and study periods. The lack of consensus in the definition of MCI has imposed serious challenges on previous reviews [25]. Different diagnostic confirmation tools can result in different MCI prevalence results [116]. The Petersen method [11] is based on four criteria: subjective memory complaint, objective memory disorder, normal functional activities, and absence of dementia. In contrast, the NIA-AA [103] allows inclusion of MRI imaging and cerebrospinal fluid tests as evidence, boosting the chance of MCI detection. However, our study shows that MCI prevalence is lower in the studies using NIA-AA compared to those applying the Petersen criteria. Such a contradiction may be associated with the fact that MRI imaging instruments and biomarker tests are optional and are likely to be ignored by many studies due to resource restrictions. Adding to the complexity is the use of screening as a first step to identify MCI patients. Variations in screening instruments and cut-off thresholds can lead to different results too [117]. MMSE [76] is the most commonly used cognitive screening tool worldwide, providing a comprehensive assessment on cognitive function in seven domains. However, the MMSE lacks sensitivity to detect MCI. While MoCA [77] meets the criteria with both high sensitivity and relatively high specificity in MCI detection ( $\mathrm{Sn}=81$ 97\%; $\mathrm{Sp}=60-86 \%$ ) [118], it has been recommended as a preferred screening tool in $\mathrm{MCI}$ detection in primary care setting. It is believed that the variation of results across the study period can also be partly attributed to variations in screening diagnostic tools [119].

Differentiating between aMCI and naMCI may help address some of the above issues by offering greater clarity in selecting diagnostic and screening tools. But unfortunately, only a small percentage of studies chose to do so, perhaps because additional cognitive domains such as language, vision, and listening need to be assessed. It is important to note that the DSM-IV diagnostic confirmation method, used in 9 included studies in this systematic review, detects amnesic cognitive disorders and could underestimate the overall prevalence of MCI [120]. But it does not seem to be the case. This is likely to be a result of confounding effects of different screening tools.
Our sub-group meta-analyses revealed that MCI prevalence increases with age. Women, rural residents, and those who live alone and have low levels of education are likely to have higher MCI prevalence than others.

Aging has been reported as the most common risk factor for MCI [121]. Our study provides further evidence to support this argument. The prevalence of MCI in those aged between 70 and 79 years (14.6\%) nearly doubles that of those aged between 55 and 59 years. The mechanism underling this age connection may be associated with increased oxidative stress and amyloidal accumulation in the brain [122].

In this study, we found that women are more likely to have MCI than men. This finding is consistent with results of previous systematic reviews on Chinese populations [21, 22]. This study showed, for the first time, that the same sex difference also exists in aMCI for Chinese populations, similar to that in other populations [123, 124]. Some researchers argued that hormone changes may explain the sex difference in MCI because there is evidence that hormone-replacement therapy can protect against dementia [125]. But the evidence is weak and indirect. There are studies reporting insignificant sex difference in MCI [126], or even higher prevalence of MCI in older adult men [115].

Socioeconomic disparities in MCI prevalence deserve increasing academic and policy attention. This systematic review confirmed that low levels of education can exacerbate the occurrence of cognitive impairment, including dementia as concluded in some other studies [127-129]. This is unlikely a result of screening or diagnostic bias as all neuropsychological tests have been corrected for education. Some researchers believe that education can enhance the brain's ability to make efficient use of cognitive networks [130,131]. Furthermore, those who live alone are more likely to have MCI. This may be associated with a lack of communication, anxiety, and depression [132]. The urban-rural difference in MCI prevalence may have some unique implications for China. Although it appears to be an international phenomenon with rural residents having higher MCI prevalence than their urban counterparts [133], possibly due to increased health risks and chronic conditions (such as diabetes and hypertension) [134-136], China's dual welfare systems present some particular challenges for addressing the problem. Rural residents in mainland China usually have lower income, live in poorer housing conditions, receive less education, and enjoy lower levels of social and health entitlements compared with the urban ones. During the dramatic transition period with unprecedented economic development, a large proportion of young rural residents moved to urban centres for better education and job opportunities, leaving their 
older family members alone at home. A combination of these risk factors can expose rural older residents in a serious vulnerable position to cognitive impairment and dementia [137].

High levels of heterogeneity were observed for the pooled analysis of $\mathrm{MCI}$ and $\mathrm{aMCI}$, as well as for the sub-group analysis of MCI. Although we adopted the recommended methods for handling the heterogeneity, it is noteworthy when applying the findings of this study. Indeed, previous studies reported an increase of MCI prevalence in China from 5\% in 2000 to about $20 \%$ in 2014 [22]. This may be a result of several underlined reasons. We found in this study that cohort effect can explain about $21 \%$ of heterogeneity of the included studies in this systematic review. However, China has experienced dramatic socioeconomic transformation over the period. These include, but not limited to, prolonged life expectancy and arrival of an ageing society, increase in morbidity of chronic conditions such as diabetes and hypertension, and rapid advancement of medical technologies and medical care services. All of these can compound the prevalence of MCI. The true cohort effect can only be revealed through future studies using a method that can separate the effects of age, cohort, study period, and other confounding factors [138]. At this stage, the interpretation and application of the pooled results of this systematic review should be cautious. It is not unreasonable to anticipate a further increase in MCI prevalence as China continues its aging process. Local communities should consider the characteristics of their community residents in estimating local prevalence of MCI.

\section{Strengths and limitations}

This study has several strengths. Firstly, this systematic review restricted studies to those of community-dwelling noninstitutionalised residents. Very few, if any, Chinese people have received institutionalised care due to social, cultural and economic reasons. This enables better generalisability of findings to community populations. Secondly, this study included extensive literature searching for studies published in both English and Chinese languages. Thirdly, this study adopted more stringent inclusion and exclusion criteria to ensure high quality results. The analyses were based on confirmed, not suspected cases of MCI. Moreover, this systematic review involved a separate analysis on aMCI.

Despite the strengths, there are several limitations in this study. Lack of enough available data may account for the reasons why we had identified no more than one significant predictor in the meta-regression analysis. There is a shortage of studies into the subtypes of MCI, which prevented us from performing further subgroup analyses on aMCI prevalence. Only two studies $[19,20]$ included in the meta-analysis drew results from a nation-wide sample. The rest had participants from different regions. Significant socioeconomic disparities exist across regions in China. We found no study involving minority ethnicity groups. China has 56 ethnic groups. Further studies into these populations are needed. A national study using a unified protocol is preferred.

\section{Conclusion}

This study shows that $12.2 \%$ of Chinese populations over 55 years have $\mathrm{MCI}$ and $10.9 \%$ have aMCI. MCI prevalence increases with age. Women, rural residents, and those who live alone and have low levels of education have higher MCI prevalence than others. The results also vary with diagnostic criteria and study periods. Increasing attention should be paid to regional disparities in future studies as socioeconomic disparities across regions continue to grow in China.

\section{Supplementary Information}

The online version contains supplementary material available at https://doi. org/10.1186/s12877-020-01948-3.

\section{Additional file 1}

Additional file 2.

\begin{abstract}
Abbreviations
ADL: Activity of Daily Living; ALT: Associative Learning Test; AMCl: Amnesic Mild Cognitive ImpairmentAVLTAuditory Verbal Learning Test;

CAMCOG: Cambridge Cognitive Examination; CCAS: Chinese Cognitive Ability Scale; CDR: Clinical Dementia Rating Scale; CDT: Clock Drawing Test; CESD: The Center for Epidemiologic Studies Depression Scale; CSI-

D: Community Screening Instrument for Dementia; DS: Digit Span; DSMIV: the American Psychiatric Association's Diagnostic and Statistical Manual of Mental Disorder 4th edition; FAQ: Functional Activities Questionnaire; GDS: Global Deterioration Scale; GMS: Geriatric Mental State;

HAMD: Hamilton Depression Rating Scale; HIS: Hachinski Ischemic Index; IMCT: Information-Memory-Concentration test; MCl: Mild Cognitive Impairment; MMSE: Mini-Mental State Examination (MMSE); MoCA: Montreal Cognitive Assessment; NIA-AA: National Institute on Aging Alzheimer's Association (NIA-AA); NPI-Q: Neuropsychiatric Inventory Questionnaire; QCSTE: Quick Cognitive Screening Scale; ROCFR: Rey-Osterrieth Complex Figure Recall Tests; SAS: Self-Rating Anxiety Scale; SCID: Structured Clinical Interview for DSM-IV; SDMT: Symbol Digit Modalities Test; STMT: Semantic Trail Making Test; VFT: Verbal Fluency Test; WAIS: Wechsler Adult Intelligence Scale; WHOBCAl: World Health Organization-Battery of Cognitive Assessment Instrument; WHODAS-12: 12-item WHO Disability Assessment Schedule; WMS-R: Wechsler Memory Scale-Revised
\end{abstract}

\section{Acknowledgements}

The authors thank the faculty members of Statistics Consultant Xia Li, La Trobe University of Mathematics \& Statistics.

\section{Authors' contributions}

YL, CL1 and DY conceived and designed the study. YL collected and analysed the data, prepared figures/tables, contributed drafts of the manuscript. CL1, DY and SF critically reviewed the contents and modified the draft. JM and MZ participated in collecting and analysing the data. CL2 provided data analysis support. All authors read and approved the final manuscript.

\section{Funding}

The project was supported by the fund from Shanghai Municipal Health Commission, Shanghai, China (201940495). This work was also supported by the Australian Government Research Training Program Fees Offset (RTP Fees 
Offset) and the La Trobe University Full Fee Research Scholarship (LTUFFRS). The funding bodies do not have any involvement in the design, execution and writing of the study.

\section{Availability of data and materials}

All data generated and analysed during this study are included in this manuscript and the supporting file.

\section{Ethics approval and consent to participate}

Not applicable.

\section{Consent for publication}

Not applicable.

\section{Competing interests}

The authors declare there are no conflicts of interest for this study.

\section{Author details}

'Department of General Practice, Yangpu hospital, Tongji University School of Medicine, Shanghai 200090, China. ${ }^{2}$ School of Psychology and Public Health, La Trobe University, Melbourne, VIC 3086, Australia. ${ }^{3}$ Academic Department of General Practice, Yangpu hospital, Tongji University School of Medicine, Shanghai 200090, China. ${ }^{4}$ Shanghai General Practice and Community Health Development Research Center, 200090, Shanghai, China. ${ }^{5}$ Clinical Research Center, Shanghai Mental Health Center, Shanghai Jiao Tong University School of Medicine, Shangha, China.

Received: 22 June 2020 Accepted: 6 December 2020

Published online: 06 January 2021

\section{References}

1. World Alzheimer Report 2016: Improving healthcare for people living with dementia:coverage, quality and costs now and in the future. Available online: https://www.alz.co.uk/research/world-report-2016 (accessed on 24 November 2016).

2. Alwan A, Maclean DR, Riley LM, d'Espaignet ET, Mathers CD, Stevens GA, et al. Monitoring and surveillance of chronic non-communicable diseases: progress and capacity in high-burden countries. Lancet. 2010;376(9755): 1861-8.

3. Xu J, Wang J, Wimo A, Fratiglioni L, Qiu C. The economic burden of dementia in China, 1990-2030: implications for health policy. Bull World Health Organ. 2017;95(1):18-26.

4. Jia JP, Wei CB, Chen SQ, Li FY, Tang Y, Qin W, et al. The cost of Alzheimer's disease in China and re-estimation of costs worldwide. Alzheimers Dement. 2018;14(4):483-91.

5. Livingston G, Sommerlad A, Orgeta V, Costafreda SG, Huntley J, Ames D, et al. Dementia prevention, intervention, and care. Lancet. 2017;390(10113):2673-34.

6. Petersen RC. Normal aging, mild cognitive impairment, and early Alzheimer's disease. Neurologist. 1995;1 (6):326-44.

7. Winblad B, Palmer K, Kivipelto M, Jelic V, Fratiglioni L. O. Wahlund L, et al. MCl toward a consensus. J Intern Med. 2004;256:240-6.

8. Morris JC, Cummings J. Mild cognitive impairment (MCI) represents earlystage Alzheimer's disease. J Alzheimers Dis. 2005;7(3):235-62.

9. Busse A, Angermeyer MC, Riedel-Heller SG. Progression of mild cognitive impairment to dementia: a challenge to current thinking. Br J Psychiatry. 2006;189:399-404

10. Gauthier S, Reisberg B, Zaudig M, Petersen RC, Ritchie K, Broich K, et al. Mild cognitive impairment. Lancet. 2006;367(9518):1262-70.

11. Petersen RC. Mild cognitive impairment as a diagnostic entity. J Intern Med. 2004;256(3):183-94.

12. Petersen RC, Roberts RO, Knopman DS, Boeve BF, Geda YE, Ivnik RJ, Smith GE, Jack CR Jr. Mild cognitive impairment: ten years later. Arch Neurol. 2009; 66(12):1447-55.

13. Ward A, Arrighi HM, Michels S, Cedarbaum JM. Mild cognitive impairment: disparity of incidence and prevalence estimates. Alzheimers Dement. 2012; 8(1):14-21.

14. Petersen RC, Lopez O, Armstrong MJ, Getchius TSD, Ganguli M, Gloss D, et al. Practice guideline update summary: mild cognitive impairment: Report of the guideline development, dissemination, and implementation Subcommittee of the American Academy of neurology. Neurology. 2018; 90(3):126-35.
15. Lara E, Koyanagi A, Olaya B, Lobo A, Miret M, Tyrovolas S, et al. Mild cognitive impairment in a Spanish representative sample: prevalence and associated factors. Int J Geriatr Psychiatry. 2016;31(8):858-67.

16. Brucki SMD. Epidemiology of mild cognitive impairment in Brazil. Dement Neuropsychol. 2013;7(4):363-6.

17. Alkhunizan M, Alkhenizan A, Basudan L. Prevalence of mild cognitive impairment and dementia in Saudi Arabia: a community-based study. Dement Geriatr Cogn Dis Extra. 2018;8(1):98-103.

18. Bae S, Shimada H, Lee S, Makizako H, Lee S, Harada K, et al. The relationships between components of metabolic syndrome and mild cognitive impairment subtypes: a cross-sectional study of Japanese older adults. J Alzheimers Dis. 2017;60(3):913-21.

19. Sosa AL, Albanese E, Stephan BC, Dewey M, Acosta D, Ferri CP, et al. Prevalence, distribution, and impact of mild cognitive impairment in Latin America, China, and India: a 10/66 population-based study. PLoS Med. 2012; 9(2):e1001170

20. Jia J, Zhou A, Wei C, Jia XF, Wang F, Li F, et al. The prevalence of mild cognitive impairment and its etiological subtypes in elderly Chinese. Alzheimers Dement. 2014;10(4):439-47.

21. Nie H, Xu Y, Liu B, Zhang YD, Lei T, Hui XP, et al. The prevalence of mild cognitive impairment about elderly population in China: a meta-analysis. Int J Geriatr Psychiatry. 2011;26(6):558-63.

22. Xue J, Li J, Liang JM, Chen SL. The prevalence of mild cognitive impairment in China: a systematic review. Aging Dis. 2018;9(4):706-15.

23. Yang $L$, Qin QB. Study on prevalence and risk factors of mild cognitive impairment among retired cadres. Chin J Nervous Mental Dis. 2011;37(8):473-6.

24. Qiu CJ, Tang MN, Zhang W, Han HY, Dai J, Lu J, et al. The prevalence of mild cognitive impairment among residents aged 55 or over in Chengdu area. Chin J Epidemiol. 2003;24(12):1104-7.

25. Lee SB, Kim KW, Youn JC, Park JH, Lee JJ, Kim MH, et al. Prevalence of mild cognitive impairment and its subtypes are influenced by the application of diagnostic criteria: results from the Korean longitudinal study on health and aging (KLoSHA). Dement Geriatr Cogn Disord. 2009;28(1):23-9.

26. Bondi MW, Smith GE. Mild cognitive impairment: a concept and diagnostic entity in need of input from neuropsychology. J Int Neuropsychol Soc. 2014;20(2):129-34.

27. Kaur J, Sidhu B, Sibia R, Kaur B. Prevalence of mild cognitive impairment among hospital Patiens aged 65 and above. J Delhi Psychiatry J. 2014;17(1):60-4.

28. Ni HJ, Qin JL, Zhou LP, Zhao ZG. Wang J, Hou FZ, for the Alzheimer's Disease Neuroimaging Initiative (ADNI). Network analysis in detection of early-stage mild cognitive impairment. Physica A-statistical Mechanics Applications. 2017:478:113-9.

29. Hoy D, Brooks P, Woolf A, Blyth F, March L, Bain C, et al. Assessing risk of bias in prevalence studies: modification of an existing tool and evidence of interrater agreement. J Clin Epidemiol. 2012;65(9):934-9.

30. Guyatt G, Oxman AD, Akl EA, Kunz R, Vist G, Brozek J, et al. GRADE guidelines: 1. Introduction-GRADE evidence profiles and summary of findings tables. J Clin Epidemiol. 2011;64(4):383-94.

31. Janoutova J, Ambroz P, Kovalova M, Machaczka O, Němček K, Zatloukalová A, et al. Epidemiology of mild cognitive impairment. Ceska a Slovenska Neurologie a Neurochirurgie. 2018;81(3):284-9.

32. Egger M, Davey Smith G, Schneider M, Minder C. Bias in meta-analysis detected by a simple, graphical test. BMJ. 1997;315(7109):629-34.

33. Higgins JP, Thompson SG, Deeks JJ, Altman DG. Measuring inconsistency in meta-analyses. BMJ. 2003;327(7414):557-60.

34. Lipsey MW, Wilson DB. Practical meta-analysis. Thousand Oaks: Sage Publications; 2001. p. 727-8.

35. Chen ND, Du J, Yang Y, Li D, Xu LB, Li W, et al. Comparation of two diagnostic criteria on mild cognitive impairment in an elderly cohort. J Neurosci Mental Health. 2014;14(3):247-50.

36. Ding D, Zhao QH, Guo QH, Meng HJ, Wang B, Luo JF, et al. Prevalence of mild cognitive impairment in an urban community in China: a cross-sectional analysis of the Shanghai Aging Study. Alzheimers Dement. 2015;11(3):300-9.e2.

37. Guo GY, Tan GY, Zhang SF, Yu XD, Li CL, et al. Status analysis of mild cognitive im pairment of the elderly in Chengde City. China Medical Herald. 2013;10(6):110-1.

38. Guo XY, Zhao LM, Li XM, Yang Q, et al. Prevalence of mild cognitive impairment among rural Chinese elderly. Chin J Mult Organ Dis Elderly. 2013;12(12):904-7.

39. Hai S, Dong BR, Liu YX, Zou YP. Occurrence and risk factors of mild cognitive impairment in the older Chinese population: a 3-year follow-up study. Int J Geriatr Psychiatry. 2012;27(7):703-8. 
40. He L. Assessment and intervention study of mild cognitive impairment in the elderly in community of Jiangxi Province [D]. NanChang University; 2015.

41. Hu R, Zhao SG, Wang DS, Wen SR, Niu GM. A Rong, et al. a prevalence study on mild cognitive impairment among the elderly populations of Mongolian and Han nationalities in a pastoral area of inner Mongoli a. Chin J Epidemiol. 2012;33(4):364-7

42. Huang R, Tang MN, Ma C, Guo YB, Han HY, Huang JM, et al. The prevalence of mild cognitive impairment of residents aged 60 years and over in the urban and rural areas in Guangzhou. Chin J Nerv Ment Dis. 2008;34(9):533-7.

43. Jiang $L J$. The follow-up study of assessment, classification, and intervention of the cognitive impairment in the elderly in the Changchun south lake community [D]. JiLin University; 2017.

44. Lao ML, Zhang HY, Luo G, Yi XN, Huang YD, Wu ZH, et al. Prevalence of mild cognitive impairment amony 55-years old or over individuals in Hainan Island. Hainan Med J. 2011;22(14):112-4.

45. Li CP, Wang Y, Tian F, Liu X, Liang YJ, Zhang WX, et al. Prevalence and risk factors of mild cognitive impairment among rural Chinese elderly. Chin J Gerontol. 2015;35:3404-7.

46. Li W, Sun L, Xiao SF. Prevalence, incidence, influence factors, and cognitive characteristics of amnestic mild cognitive impairment among older adult: a 1-year follow-up study in China. Front Psychiatry. 2020;11:75.

47. Li X, Ma C, Zhang J, Liang Y, Chen YJ, Chen KW, et al. Prevalence of and potential risk factors for mild cognitive impairment in community-dwelling residents of Beijing. J Am Geriatr Soc. 2013;61(12):2111-9.

48. Liao B, Gao R, Xiong LH, Yi GP, Sheng ZJ, Liu MG, et al. The Early Evaluation and Intervention Strategies of Mild Cognitive Impairment in Yichun Area. J Yichun College. 2012;34(12):77-9.

49. Liu H, Zeng QZ, Zhuang XW, Chen YM. Investigation analysis of mild cognitive impairment of the elderly in the community of some District in Shanghai and study of the intervention methods and effects. J Int Psychiatry. 2018;45(2):288-91,301.

50. Ma F, Wu TF, Zhao JG, Li L, Song AL, Zhang ML, et al. Prevalence of mild cognitive impairment and its subtypes among Chinese older adults: role of vascular risk factors. Dement Geriatr Cogn Disord. 2016;41(5-6):261-72.

51. Meng WQ. Cigarette smoking and alcohol consumption in relation to mild cognitive impairment in old people in Inner Mongolia [D]. Inner Mongolia University; 2010

52. Pan ZD, Zhou RS, Wang T, Xian SF. Prevalence of mild cognitive impairment among the elderly in community. Geriatr Health Care. 2012;18(3):154-6.

53. Qin HY, Chen DH, Qu ZW. Investigation of mild cognitive impairment and its risk factors among 55 years old and above residents in Shanghai. J Clin Psychiatry. 2014;24(3):155-8.

54. Rao DP, Luo X, Tang MN, Shen Y, Huang RY, Yu JC, et al. Prevalence of mild cognitive impairment and its subtypes in community-dwelling residents aged 65 years or older in Guangzhou. China Arch Gerontol Geriatr. 2018;75:70-5.

55. Ren CF, Yuan YF, Zhang Y, Wang JL, Lu HL, Wan AL, et al. Analysis on prevalence and influencing factors of mild cognitive impairment among the elderly in Jiangxi Communitie. Modem Prev Med. 2013;40(21):3969-73.

56. Song $X Z$, Chen $J H, H e ~ L P$. Investigation on correlation between prevalence of the mild cognitive impairment and eating habit in elderly in the communities of Shunde city. IMHGN. 2012;18(12):1715-8.

57. Su C. Cognitive features and normal elderly cognitive function change rule among residents aged 60 years and over in Guangzhou city [D]. Guangzhou University; 2010

58. Sun Y, Lee HJ, Yang SC, Chen TF, Lin KN, Lin CC, et al. A nationwide survey of mild cognitive impairment and dementia, including very mild dementia, in Taiwan. PLoS One. 2014;9(6):e100303 Published 2014 Jun 18.

59. Tang MN, Liu XH, Yun Y, Han HY, Tang MM, Ren H, et al. A follow-up study ofmild cognitive impairment and senile dementia in the urban and rura aged population in Chengdu. Chin J Psychiatry. 2000;33(4):218-21.

60. Tang Z, Zhang XQ, Wu XG, Liu HJ, Diao LJ, Guan SC, et al. Prevalence of the Mild Cognitive Impairment among Elderly in Beijing. Chin Ment Health J. 2007;21(2):116-8

61. Wang T, Xiao SF, Chen KW, Yang CC, Dong SH, Cheng Y, et al. Prevalence, incidence, risk and protective factors of amnestic mild cognitive impairment in the elderly in Shanghai. Curr Alzheimer Res. 2017;14(4):460-6.

62. Wang T, Cao C, Deng J, Lian JX, Yan K, Wang ZZ, et al. Prevalence and risk factors of mild cognitive impairment among old people in Chongqing. Chin J Rehabil Theory Pract. 2017;23(7):833-8.
63. Wang YP, Zhai JB, ZHU F, Zhang WW, Yang XJ, Qu CY, et al. A three-year follow-up study on the transfer of mild cognitive impairment to Alzheimer's disease among the elderly in Taiyuan city. Chin J Epidemiol. 2011;32(2):105-9.

64. Wang ZZ, Ding L, Liu L, Li T, Ma WR, Zhang J L, et al. The status of mild cognitive impairment in the Hui and Han people aged 55 years and above in Ningxia. Chin J Gerontol. 2013;36:4601-3.

65. Wu Y, Yao JJ, Chen ZH, Yang QP, Zhou DX, Feng W, et al. Survey on mild cognitive impairment and analysis of its influencing factors among community elderly in Wuxi City. Modern Prev Med. 2017;44(2):259-63.

66. Xiao SF, Lewis M, Mellor D, McCabe M, Byrne L, Wang T, et al. The China longitudinal ageing study: overview of the demographic, psychosocial and cognitive data of the Shanghai sample. J Ment Health. 2016;25(2):131-6.

67. Xu SJ, Xie B, Song M, Yu LL, Wang L, An CX, et al. High prevalence of mild cognitive impairment in the elderly: a community-based study in four cities of the Hebei province, China. Neuroepidemiology. 2014:42(2):123-30.

68. Yin LY. Investigation and diagnosis of mild cognitive impairment [D].HeBei University; 2010.

69. Yuan J, Qu ZW, Jiang Q, Yang ZD, Fu WZ, et al. The epidemiological survey of depression disorder and cognitive disorder of the elderly in Shanghai Pudong community. J Chin Psychiatry. 2013;23(2):86-8.

70. Zhang $X Q, H u Z$. Prevalence and factors associated with mild cognitive impairment among the elderly in Changsha communities. Chin Gen Pract. 2014;17(9):1031-5.

71. Zhou DS, Xu YE, Chen ZM, Hu ZY, Chen YF. Prevalence of mild cognitive impairment among the elderly. Chin J Public Health. 2011;27(11):1375-7.

72. Zhu XQ, Zhou XH, Kumusi B, Yue YH, Zhao RJ, Xin SF, et al. Study of prevalence of the mild cognitive impairment among elderly in the communities of Urumqi city. J Xinjiang Med Univ. 2009;32(5):578-84.

73. Huppert FA, Brayne C, Gill C, Paykel ES, Beardsall L. CAMCOG--a concise neuropsychological test to assist dementia diagnosis: socio-demographic determinants in an elderly population sample. Br J Clin Psychol. 1995;34(4):529-41.

74. Cheng ZH, JR S. Theoretic framework of Chinese cognitive ability scale. Chin J Clin Psychol. 2006;14(4):340-2.

75. Hall KS, Hendrie HC, Brittain HM, Norton JA, Rodgers DD. The development of a dementia screening interview in two distinct languages. Int J Methods Psychiatr Res. 1993;3:1-28.

76. Folstein MF, Folstein SE, McHugh PR. "mini-mental state". A practical method for grading the cognitive state of patients for the clinician. J Psychiatr Res. 1975;12(3):189-98.

77. Nasreddine ZS, Phillips NA, Bédirian V, Charbonneau S, Whitehead V, Collin I, et al. The Montreal Cognitive Assessment, MoCA: a brief screening tool for mild cognitive impairment [published correction appears in J Am Geriatr Soc. 2019 Sep;67(9):1991]. J Am Geriatr Soc. 2005;53(4):695-9.

78. Kaufer DI, Cummings $J$, Ketchel P, et al. Validation of the NPI-Q, a brief clinical form of the neuropsychiatric inventory. J Neuropsychiatry Clin Neurosci. 2000;12(2):233-9.

79. Wu Y, Xu WW, Zao HC. Quick cognitive screening scale for the elder: development,reliability and validity. Chin J Behav Med \& Brain Sci. 2013;12(22):112932.

80. ASSOCIATION A P. Diagnostic and Statistical Manual of Mental Disorders. 4th ed. Washington, D.C.: American Psychiatric Association; 1994.

81. Bright $P$, Hale E, Gooch VJ, Myhill T, van der Linde I. The National Adult Reading Test: restandardisation against the Wechsler adult intelligence scale-fourth edition. Neuropsychol Rehabil. 2018;28(6):1019-27.

82. Liu YY, Wang T, Li X, Li GJ, Su N, Xiao SF. Reliability and validity of the Chinese version of neuropsychological test battery. Chin J Clinicians. 2011:5(5):1339-45.

83. Ustun TB, Kostanjsek N, Chatterji S, Rehm J. Measuring Health and Disability: Manual for WHO Disability Assessment Schedule (WHODAS 2.0). Geneva: World Health Oragnization,In press; 2010.

84. Wang P, Li J, Li H, Zhang S. Differences in learning rates for item and associative memories between amnestic mild cognitive impairment and healthy controls. Behav Brain Funct. 2013;9(1):29.

85. Blessed G, Tomlinson BE, Roth M. The association between quantitative measures of dementia and of senile change in the cerebral grey matter of elderly subjects. Br J Psychiatry. 1968;114(512):797-811.

86. Wechsler D. Wechsler memory scale-revised manual. San Antonio: The Psychological Corporation; 1987.

87. Sheridan LK, Fitzgerald HE, Adams KM, Nigg JT, Martel MM, Puttler LI, et al. Normative symbol digit modalities test performance in a community-based sample. Arch Clin Neuropsychol. 2006;21(1):23-8.

88. Reitan RM. Validity of the trail making test as an indicator of organic brain damage. Percept Mot Skills. 1958;8:271-6. 
89. Wolf-Klein GP, Silverstone FA, Levy AP, Brod MS. Screening for Alzheimer's disease by clock drawing. J Am Geriatr Soc. 1989;37(8):730-4.

90. Tupler LA, Welsh KA, Asare-Aboagye Y, Dawson DV. Reliability of the ReyOsterrieth complex figure in use with memory-impaired patients. J Clin Exp Neuropsychol. 1995;17(4):566-9.

91. Maj M, Satz P, Janssen R, Zaudig M, Starace F, D'Elia L, et al. WHO neuropsychiatric AIDS study, cross-sectional phase II. Neuropsychological and neurological findings. Arch Gen Psychiatry. 1994;51(1):51-61.

92. Lezak MD. Neuropsychological assessment. 5th ed. New York: Oxford University Press; 2012.

93. Katz S, Ford AB, Moskowitz RW, Jackson BA, Jaffe MW. Studies of illness in the aged. the index of ADL: a standardized measure of biological and psychosocial function. JAMA. 1963;185:914-9.

94. Pfeffer Rl, Kurosaki TT, Harrah CH Jr, Chance JM, Filos S. Measurement of functional activities in older adults in the community. J Gerontol. 1982;37(3):323-9.

95. Morris JC. The clinical dementia rating (CDR): current version and scoring rules. Neurology. 1993:43(11):2412-4.

96. Lewinsohn PM, Seeley JR, Roberts RE, Allen NB. Center for Epidemiologic Studies Depression Scale (CES-D) as a screening instrument for depression among community-residing older adults. Psychol Aging. 1997;12(2):277-87.

97. Leung JL, Lee GT, Lam YH, Chan RC, Wu JY. The use of the digit span test in screening for cognitive impairment in acute medical inpatients. Int Psychogeriatr. 2011;23(10):1569-74.

98. Hamilton M. A rating scale for depression. J Neurol Neurosurg Psychiatry. 1960;23:56-62

99. Reisberg B, Ferris SH, de Leon MJ, Crook T. The global deterioration scale for assessment of primary degenerative dementia. Am J Psychiatry. 1982;139(9):1136-9.

100. Copeland JR, Dewey ME, Griffiths-Jones HM. A computerized psychiatric diagnostic system and case nomenclature for elderly subjects: GMS and AGECAT. Psychol Med. 1986;16(1):89-99.

101. Zung WW. A rating instrument for anxiety disorders. Psychosomatics. 1971 12(6):371-9.

102. Hachinski VC, lliff LD, Zilhka E, Boulay GH, McAllister VL, Marshall J. Cerebral Blood Flow in Dementia. Arch Neurol. 1975;32(9):632-7.

103. Albert MS, DeKosky ST, Dickson D, Dubois B, Feldman HH, Fox NC, et al. The diagnosis of mild cognitive impairment due to Alzheimer's disease: recommendations from the National Institute on Aging-Alzheimer's Association workgroups on diagnostic guidelines for Alzheimer's disease. Alzheimers Dement. 2011;7(3):270-9.

104. Pan HY, Wang JQ, Wu ML, Chen JJ. Study on prevalence rate and quality of life of elderly patients with mild cognitive impairment in community. Nurs J Chin PLA. 2012;29(1B):6-9,27.

105. Peng ZR, Jiang $H$, Wang $X M$, Huang KY, Zuo YK, Wu XM, et al. The Efficacy of Cognitive Training for Elderly Chinese Individuals with Mild Cognitive Impairment. Biomed Res Int. 2019;2019:4347281-Published 2019 Nov 30

106. Wu L, He Y, Jiang B, Liu M, Wang JH, Yang SS, et al. The association between the prevalence, treatment and control of hypertension and the risk of mild cognitive impairment in an elderly urban population in China. Hypertens Res. 2016;39(5):367-75.

107. Zhong SY, Tong ZD, Hu BX, Yuan XB, Chen W, Zhang FN, et al. Prevalence and influencing factors of mild cognitive impairment among the elderly in Zhoushan City. Prev Med. 2018;30(2):170-2.

108. Vlachos GS, Kosmidis MH, Yannakoulia M, Dardiotis E, Hadjigeorgiou G, Sakka $\mathrm{P}$, et al. Prevalence of mild cognitive impairment in the elderly population in Greece: results from the HELIAD study. Alzheimer Dis Assoc Disord. 2020;34(2):156-62.

109. Janelidze M, Mikeladze N, Bochorishvili N, Dzagnidze A, Kapianidze M, Mikava N, et al. Mild Cognitive Impairment in Republic of Georgia. Gerontol Geriatr Med. 2018:4:2333721418771408 Published 2018 May 4

110. Hänninen T, Hallikainen M, Tuomainen S, Vanhanen M, Soininen $H$. Prevalence of mild cognitive impairment: a population-based study in elderly subjects. Acta Neurol Scand. 2002;106(3):148-54.

111. Tognoni G, Ceravolo R, Nucciarone B, Bianchi F, Dell'Agnello G, Ghicopulos I, et al. From mild cognitive impairment to dementia: a prevalence study in a district of Tuscany, Italy. Acta Neurol Scand. 2005;112(2):65-71.

112. Juarez-Cedillo T, Sanchez-Arenas R, Sanchez-Garcia S, Garcia-Peña C, Hsiung GY, Sepehry A, et al. Prevalence of mild cognitive impairment and its subtypes in the Mexican population. Dement Geriatr Cogn Disord. 2012;34(5-6):271-81.

113. Moretti F, De Ronchi D, Palmer K, Forlani C, Morini V, Ferrari B, et al. Prevalence and characteristics of mild cognitive impairment in the general population. Data from an Italian population-based study: the Faenza project. Aging Ment Health. 2013;17(3):267-75.

114. Henao-Arboleda E, Aguirre-Acevedo DC, Munoz C, Pineda DA, Lopera F. Prevalence of mild cognitive impairment, amnestic-type, in a Colombian population. Rev Neurol. 2008;46(12):709-13.

115. Petersen RC, Roberts RO, Knopman DS, Geda YE, Cha RH, Pankratz VS, et al. Prevalence of mild cognitive impairment is higher in men. The Mayo Clinic study of aging. Neurology. 2010;75(10):889-97.

116. Busse A, Bischkopf J, Riedel-Heller SG, Angermeyer MC. Mild cognitive impairment: prevalence and incidence according to different diagnostic criteria. Results of the Leipzig longitudinal study of the aged (LEILA75+). Br J Psychiatry. 2003;182:449-54.

117. Brodaty H, Heffernan M, Kochan NA, Draper B, Trollor JN, Reppermund S, et al. Mild cognitive impairment in a community sample: the Sydney Memory and Ageing Study. Alzheimers Dement. 2013:9(3):310-17.e1.

118. Abd Razak MA, Ahmad NA, Chan YY, Mohamad Kasim N, Yusof M, Abdul Ghani MKA, et al. Validity of screening tools for dementia and mild cognitive impairment among the elderly in primary health care: a systematic review. Public Health. 2019;169:84-92. https://doi.org/10.1016/j.puhe.

119. Chan KY, Wang W, Wu JJ, Liu L, Theodoratou E, Car J, et al. Global Health epidemiology reference group (GHERG). Epidemiology of Alzheimer's disease and other forms of dementia in China, 1990-2010: a systematic review and analysis. Lancet. 2013;381(9882):2016-23.

120. Petersen RC, O'brien J. Mild cognitive impairment should be considered for DSM-V [M]. Diagnostic issues in dementia: Advancing the research agenda for DSM-V. Arlington: American Psychiatric Association; US; 2007. p. 51-65.

121. Brookmeyer $\mathrm{R}$, Abdalla $\mathrm{N}$, Kawas $\mathrm{CH}$, Corrada MM. Forecasting the prevalence of preclinical and clinical Alzheimer's disease in the United States. Alzheimers Dement. 2018;14(2):121-9.

122. Ovod V, Ramsey KN, Mawuenyega KG, Bollinger JM, Hicks T, Schneider T, et al. Amyloid $\beta$ concentrations and stable isotope labeling kinetics of human plasma specific to central nervous systemamyloidosis [published correction appears in Alzheimers Dement. 2017 Oct;13(10):1185]. Alzheimers Dement. 2017;13(8):841-9.

123. Au B, Dale-McGrath S, Tierney MC. Sex differences in the prevalence and incidence of mild cognitive impairment: a meta-analysis. Ageing Res Rev. 2017:35:176-99.

124. Gavrila D, Antúnez C, Tormo MJ, Carles R, García Santos JM, Parrilla G, et al Prevalence of dementia and cognitive impairment in southeastern Spain: the Ariadna study. Acta Neurol Scand. 2009;120(5):300-7.

125. LeBlanc ES, Janowsky J, Chan BK, Nelson HD. Hormone replacement therapy and cognition: systematic review and meta-analysis. JAMA. 2001;285(11):1489-99.

126. Solfrizzi V, Panza F, Colacicco AM, D'Introno A, Capurso C, Torres F, et al. Italian longitudinal study on aging working group. Vascular risk factors, incidence of $\mathrm{MCl}$, and rates of progression to dementia. Neurology. 2004; 63(10):1882-91.

127. Afgin AE, Massarwa M, Schechtman E, Israeli-Korn SD, Strugatsky R, Abuful A, et al. High prevalence of mild cognitive impairment and Alzheimer's disease in arabic villages in northern Israel: impact of gender and education. J Alzheimers Dis. 2012;29(2):431-9.

128. Barnes DE, Yaffe $K$. The projected effect of risk factor reduction on Alzheimer's disease prevalence. Lancet Neurol. 2011;10(9):819-28.

129. Plassman BL, Williams JW Jr, Burke JR, Holsinger T, Benjamin S. Systematic review: factors associated with risk for and possible prevention of cognitive decline in later life. Ann Intern Med. 2010;153(3):182-93.

130. Meng X, D'Arcy C. Education and dementia in the context of the cognitive reserve hypothesis: a systematic review with meta-analyses and qualitative analyses. PLoS One. 2012;7(6):e38268.

131. Pettigrew C, Soldan A. Defining Cognitive Reserve and Implications for Cognitive Aging. Curr Neurol Neurosci Rep. 2019;19(1):1 Published 2019 Jan 9.

132. Ismail Z, Elbayoumi H, Fischer CE, Hogan DB, Millikin CP, Schweizer T, et al. Prevalence of depression in patients with mild cognitive impairment: a systematic review and meta-analysis. JAMA Psychiatry. 2017:74(1):58-67.

133. Nunes B, Silva RD, Cruz VT, Roriz JM, Pais J, Silva MC. Prevalence and pattern of cognitive impairment in rural and urban populations from Northern Portugal. BMC Neurol. 2010;10:42 Published 2010 Jun 11.

134. Beydoun MA, Beydoun HA, Gamaldo AA, Teel A, Zonderman AB, Wang $Y$. Epidemiologic studies of modifiable factors associated with cognition and 
dementia: systematic review and meta-analysis. BMC Public Health. 2014;14: 643 Published 2014 Jun 24

135. Ng TP, Feng L, Nyunt MS, Feng L, Gao Q, Lim ML, Collinson SL, Chong MS, et al. Metabolic syndrome and the risk of mild cognitive impairment and progression to dementia: follow-up of the Singapore longitudinal ageing study cohort. JAMA Neurol. 2016;73(4):456-63.

136. Pal K, Mukadam N, Petersen I, Cooper C. Mild cognitive impairment and progression to dementia in people with diabetes, prediabetes and metabolic syndrome: a systematic review and meta-analysis. Soc Psychiatry Psychiatr Epidemiol. 2018;53(11):1149-60.

137. Zhang X, Dupre ME, Qiu L, Zhou W, Zhao Y, Gu D. Urban-rural differences in the association between access to healthcare and health outcomes among older adults iOn China. BMC Geriatr. 2017;17(1):151 Published 2017 Jul 19.

138. Sun Y, Chang J, Liu X, Liu C. Mortality trends of liver diseases in mainland China over three decades: an age-period-cohort analysis. BMJ Open. 2019;9: e029793 doi:10.1136/ bmjopen-2019-029793.

\section{Publisher's Note}

Springer Nature remains neutral with regard to jurisdictional claims in published maps and institutional affiliations.

Ready to submit your research? Choose BMC and benefit from:

- fast, convenient online submission

- thorough peer review by experienced researchers in your field

- rapid publication on acceptance

- support for research data, including large and complex data types

- gold Open Access which fosters wider collaboration and increased citations

- maximum visibility for your research: over $100 \mathrm{M}$ website views per year

At BMC, research is always in progress.

Learn more biomedcentral.com/submissions 